Document downloaded from:

http://hdl.handle.net/10251/63825

This paper must be cited as:

Rovira Más, F.; Chatterjee, I.; Sáiz Rubio, V. (2015). The role of GNSS in the navigation strategies of cost-effective agricultural robots. Computers and Electronics in Agriculture. 112:172-183. doi:10.1016/j.compag.2014.12.017.

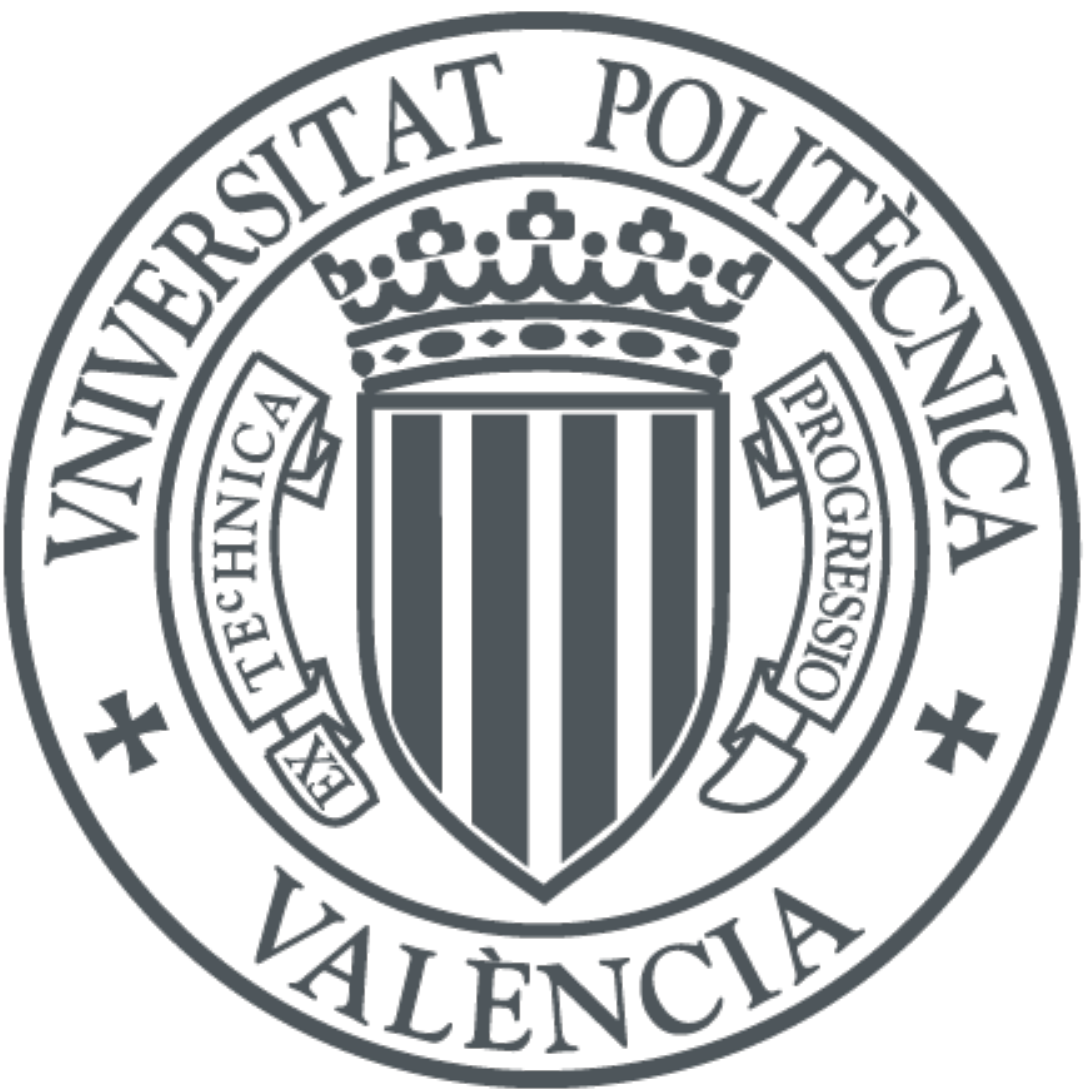

The final publication is available at

https://dx.doi.org/10.1016/j.compag.2014.12.017

Copyright Elsevier

Additional Information 
1 The role of GNSS in the navigation strategies of cost-effective

2

4

5

6

7

8

9

10

\section{agricultural robots}

\author{
Francisco Rovira-Más ${ }^{1, *}$, Ishani Chatterjee², Verónica Sáiz-Rubio ${ }^{1}$ \\ ${ }^{1}$ Agricultural Robotics Laboratory, Universidad Politécnica de Valencia, Camino de Vera s/n, 46022 \\ Valencia, Spain \\ ${ }^{2}$ Department of Agricultural and Food Engineering, Indian Institute of Technology, Kharagpur, India \\ *Corresponding author: Francisco Rovira-Más; Tel: +34 963877291; email: frovira@dmta.upv.es
}

The current state of automation technology has led to a growing interest in the design and development of new use-case agricultural robots that can provide effective solutions to the challenges that agriculture is facing in industrialized countries, especially those derived from labor shortage and ever-increasing production costs. The advent of autonomous moderate-size machines in the field appears as a prospective way of promoting the sustainable production of food in Europe, Japan, and North America. However, there exist important obstacles to the broad expansion of autonomous robots in the field; reliability, safeguarding, system complexity, and cost-efficiency in particular rank high among the impediments to overcome before prototypes move into the production stage. Robot navigation is essential for the successful deployment of autonomous machines in conventional farms, as a minimum level of safety has to be granted at the same time that navigation engines cannot be too sophisticated for solutions to compete with current equipment. In such compromise, global navigation satellite systems play a key role due to its wide range of solutions, and an important number of limitations. In this research, a variety of experiments were conducted to determine the scope of GNSS solutions as a principal component of the navigation system of novel farm robots. Results showed that regardless of the quality of the receiver used, multipath and 
other uncontrollable errors eventually occur in the field, and therefore signal consistency must be continuously checked by the robot's navigation engine. Different strategies based on the meticulous analysis of NMEA strings, the optimal combination of GGA and VTG messages, and the trajectory-based redundant estimation of robot planar states are proposed to enhance the integration of GNSS measurements in the navigation engine of agricultural robots.

\section{Keywords}

Agricultural robotics; farm robots; GNSS; navigation strategies; autonomous navigation

\section{Introduction}

The accessibility of GNSS (Global Navigation Satellite Systems) for civil applications has revolutionized the way we farm today. Precision farming packages are available from most of the major manufacturers of agricultural equipment, and sitespecific management is gaining acceptance on a global scale. Global positioning systems are an essential technology for agriculture not only for research but also in practical use (Chosa et al., 2007). Therefore, disregarding global positioning systems when building the navigation strategies of field robots, especially those performing agricultural tasks, would probably lead to limited solutions and an inefficient use of available resources. As a matter of fact, nearly all robots designed for outdoor operations - except planetary rovers-incorporate some sort of global navigation, and the trend has been moving upward since the cancelation of selective availability in 2000. A study of patents on intelligent vehicles and automatic navigation for the period 1985-2008 (Rovira-Más, 2009) reveals that eighty percent of the patents related to GPS (Global Positioning System) were published after 2000. The growing interest in satellite navigation for agricultural applications has motivated the development of ASABE Standard X587 (ASABE, 2010), which focuses on performance of positioning devices subjected to ground-based 
agricultural field operations.

The easy access to GNSS information, however, contrasts with the difficulty of assuring long-term robust and reliable measurements, especially for crucial dynamic states such as heading and speed. GPS dynamic accuracy depends on the travel direction due to satellite geometry in mid-latitude areas where satellite distribution is uneven because of the lack of satellites in the northern skies (Wu et al., 2006). Specifically, substantial differences have been found between north dilution of precision (NDOP) and east DOP (EDOP), being NDOP usually higher than EDOP. Likewise, the cross-track $\mathrm{DOP}(\mathrm{XDOP})$ perpendicular to the travel direction increases as the reference axis changes from $0^{\circ}$ to $90^{\circ}$, which explains the direction dependency of cross-track errors. GPS-based velocity estimation, on the other hand, usually provides positive results as long as there are no satellite coverage discontinuities provoked by overhead structures, electromagnetic interferences onboard are kept low, and multipath signal reception is reduced as much as possible, especially reflections caused by water bodies (Cocco and Rapuano, 2007).

Given that GNSS provides useful navigation information when estimates are reliable, but reliability cannot be always granted in general terms, farm robots must be endowed with an advanced architecture in such a way that the navigation algorithms embedded in the robot always make an optimized use of available data in real time. The Robotra tillage robot developed in 1997 (Kondo et al., 2011), for example, features an architecture based on a main computer that receives GNSS navigation data through an RS-232C bus and a complementary vehicle controller that receives feedback information from the robot sensors and exerts navigational control commands to actuate the steering mechanism, the gear shift, the throttle, and the independent brakes. Safety was tackled with three fundamental actions occurring concurrently: a feeling bumper, a contactless obstacle detector, and a remote control stop switch. The navigation strategy for the 
Robotra robot consists of a pre-planned path achieved by combining an RTK-GPS, and

80 inertial measurement unit (IMU), and a geomagnetic direction sensor. The general

81 architecture for agricultural vehicles proposed by Rovira-Más (2010) considers that the intelligent behaviors embedded in farm robots may follow a three-layer classification of

83 tasks, where the task with the highest priority is safety, followed by the other two; namely,

84 the information layer and the machine actuation layer. The information layer handles all 85 the data coming from the sensors whereas the actuation layer executes orders and commands. In order to keep these three layers continuously iterating, a system

87 architecture is necessary, and four structural sub-systems are proposed as its building blocks; local perception, global localization, actuation and control, and data processing and storage. Advanced navigation, therefore, requires the proper functioning of the four

90 of them. The particular architecture developed for the Robotra tillage tractor easily fits the general architecture defined by Rovira-Más (2010). In a similar fashion, Emmi et al. (2014) propose an architectural design based on three main modules devoted to sensing, acting, and decision making, being GNSS included in the sensing module. This architecture has been successfully tested for weed control in corn fields, where an offroad vehicle was programmed to follow a pre-defined path indicated by GPS waypoints, although perception was necessary at row initiation due to the uncertainty in determining 97 the starting point.

All the architectures described above integrate GNSS as one of their main components, and the majority of field applications reported by the agricultural sector use

100 global-based data for vehicle navigation, typically combined with other sensors. Van 101 Begeijk et al. (1998), for instance, estimated heading redundantly with a GPS receiver and an electronic flux-gate compass. The compass turned out to be the weakest point in

103 the positioning system in terms of accuracy and mounting possibilities, and consequently, 
104 trajectory-based GPS heading resulted more precise than compass heading for forward

105 velocities greater than $1 \mathrm{~m} / \mathrm{s}$. Field work demonstrated the strong dependency of heading

106 calculation on traveling speed, and showed that compasses are difficult to mount on off-

107 road vehicles, as $1.5 \mathrm{~m}$ clearance from iron and steel parts is at least necessary to avoid

108 magnetic disturbances. The actuation of an autonomous mechanical weeder (Nørremark

109 et al., 2008) involved the global referencing of individual plants as well as that of the

110 weeding robot, where the forward velocity was supplied by an onboard RTK-GPS

111 receiver and the orientation of the route was directly estimated from a sequence of

112 waypoints. The strong dependence of heading estimation on traveling speed noticed by

113 Van Begeijk et al. (1998) led Anderson and Bevly (2010) to propose velocity-based

114 course measurements from the combination of a GPS and a low-cost gyroscope through

115 a Kalman-based estimator. The complexity of adding a gyroscope through a model-based

116 estimator allowed the measurement of such vehicle states as sideslip and yaw rate, in

117 addition to habitual heading and speed. Inertial sensors, however, have not been the only

118 assistance to strengthen the navigation skills of autonomous vehicles, as perception

119 sensors have resulted helpful to assure positioning data when GPS signals are attenuated

120 or blocked, as the laser-based range-domain integration of Joerger and Pervan (2006), or

121 the automatic weed detector developed by Emmi et al. (2014), which detected the center

122 of crop rows at the headland with machine vision but continued the pre-established path

123 defined by GPS waypoints.

124 The use of GNSS permeates through most of the solutions devised in the last two

125 decades for robot navigation outdoors, although a large number of them are either too

126 unreliable or too expensive for their practical integration in farm robots. Although RTK-

127 GPS provides the highly accurate positioning needed to control field machinery and farm

128 work precisely, it is too expensive to be widely used in farm machinery. A positioning 
129 system is therefore required that is less expensive and equipped to handle centimeter

130 accuracy; however, the accuracy of a system in practical use is unclear (Chosa et al.,

131 2007). Given that obtaining accurate velocity information has traditionally involved

132 expensive solutions such as optical sensors and RTK-GPS, Serrano et al. (2004) have

133 proposed estimating velocity from the time-differential method using a stand-alone

134 single-frequency receiver without resolving carrier phase ambiguities. This solution,

135 however, requires the application of post-processing software and the availability of

136 carrier and Doppler measurements, which limit the needs for real-time navigation data of

137 farm robots and complicate the practical integration of GNSS receivers in the field as

138 wave functions must be acquired and processed rather than standard GNSS messages such

139 as those shown in Fig. 1. The problems found with Serrano's approach caused by the need

140 of knowing carrier phase measurements and the receiver positions before and after each

141 epoch, which make the method unsuitable for real-time applications, were circumvented

142 by an algorithm that uses receiver position at epoch $t$ and satellite positions at epoch $t+$

$143 \Delta t$ (Ding and Wang, 2011). However, this method also uses carrier phase and wavelength

144 in its formulation as well as time ambiguity and clock biases, all of them parameters of

145 difficult access for commercial users. In addition, given that Ding and Wang based their

146 approach on single point positioning (SPP), ionosphere and troposphere models had to be

147 used to compensate raw measurements, which can be efficiently avoided by using a

148 differential receiver (DGPS), perfectly compatible with the assumption of cost-efficiency

149 held in this research. Chosa et al. (2007) proved that, after error compensation, accurate

150 positioning is possible with a stand-alone single-frequency GNSS receiver, where

151 velocity can be derived by measuring the Doppler shift of carrier waves.

152 The main objective pursued with this research is to determine the role of GNSS

153 information in the navigation strategies of agricultural robots according to the equilibrium 
154 between reliability and cost-efficiency, using standard GNSS messages as positioning

155 sensor inputs for the system integration. As a result, measurements and analyses of the

156 raw carrier waves emitted by satellites will not be considered in the algorithms and

157 solutions developed hereafter.

\section{2. Methodology}

159 When navigation strategies are to be defined, especially if local and global data

160 are going to be fused, the selection of an advantageous coordinate system is of primary

161 importance. The local tangent plane, commonly known as easting-northing, provides an

162 ideal setting for fusing vehicle states measured with sensors of diverse nature (Rovira-

163 Más et al., 2010). Table 1 correlates the most common navigation parameters used by

164 field robots with conventional sensors of widespread use in off-road vehicles. Notice the

165 degree of redundancy inherent in the table in general, as well as the particular case of

166 GNSS where some parameters can also be redundantly estimated only with GNSS

167 messages. In light of this concurrence of - a priori- equivalent measurements, some sort

168 of hierarchy needs being established before data are processed by the navigation engine

169 of the robot. The point of this paper is ranking GNSS information through Table 1 in such

170 a way that only the most reliable estimates get to the navigation algorithms. This

171 prioritization will necessarily change with the surrounding conditions, and assessments

172 must be performed iteratively in real time.

Table 1. Navigation data sources for agricultural robots.

\begin{tabular}{cccccc} 
& Position & Time & Velocity & Heading & $\begin{array}{c}\text { Attitude } \\
\text { (pitch/roll) }\end{array}$ \\
\hline \multirow{2}{*}{ GNSS } & $\begin{array}{c}\text { Direct } \\
\text { GGA string }\end{array}$ & $\begin{array}{c}\text { Direct } \\
\text { GGA } \\
\text { string }\end{array}$ & $\begin{array}{c}\text { Direct VTG st. } \\
\text { Derived seq. }\end{array}$ & $\begin{array}{c}\text { Direct VTG st. } \\
\text { Derived seq. } \\
\text { Two receivers }\end{array}$ & Two receivers \\
\hline Gyroscope/IMU & Dead reck. & -- & $\begin{array}{c}\text { Angular } \\
\text { (yaw rate) }\end{array}$ & $\begin{array}{c}\text { Through } \\
\text { 1 integration }\end{array}$ & -- \\
\hline Doppler-Radar & Dead reck. & -- & Direct & -- & -- \\
\hline
\end{tabular}




\begin{tabular}{cccccc}
\hline Compass & -- & -- & -- & Direct & -- \\
\hline $\begin{array}{c}\text { Machine } \\
\text { Vision }\end{array}$ & $\begin{array}{c}\text { Local, } \\
\text { derived } \\
\text { from features }\end{array}$ & -- & $\begin{array}{c}\text { Derived } \\
\text { Video flow }\end{array}$ & $\begin{array}{c}\text { Local, derived } \\
\text { from features }\end{array}$ & -- \\
\hline
\end{tabular}

Abbreviations: st $\rightarrow$ string; seq $\rightarrow$ sequence; reck $\rightarrow$ reckoning; IMU $\rightarrow$ Inertial Measurement Unit

Table 1 shows that Doppler-based radar is basically used to provide an

176 independent estimate of ground speed, gyroscopes and compasses are appropriate to

177 know the heading, and machine vision is practically limited to local perception, given that

178 dead reckoning is inappropriate for off-road vehicles and environments where wheel

179 slippage is usual. The capacity of GNSS, however, is much broader, and practically all

180 the parameters listed in Table 1 can be estimated - with more or less precision- from this

181 unique source. The objective is determining when each source should be given more or

182 less weight based on its real-time reliability. The use of two separate receivers in one

183 vehicle to determine yaw, pitch, and roll requires purchasing two receivers of high

184 performance such that slight changes in attitude are precisely measured. This option,

185 however, is against the cost-efficiency premise stated in this study and therefore will not

186 be taken into account at this stage. Further studies may include the estimation of pitch

187 and roll.

According to Table 1, position and time is directly transmitted by GGA NMEA

189 (National Marine Electronic Association Standard 0183) strings, whereas velocity and

190 heading are directly conveyed by VTG strings. GNSS receivers, therefore, broadcast

191 these four basic parameters in two different strings that may be recorded simultaneously.

192 Figure 1 specifies the morphology of NMEA strings for GNSS messages. 

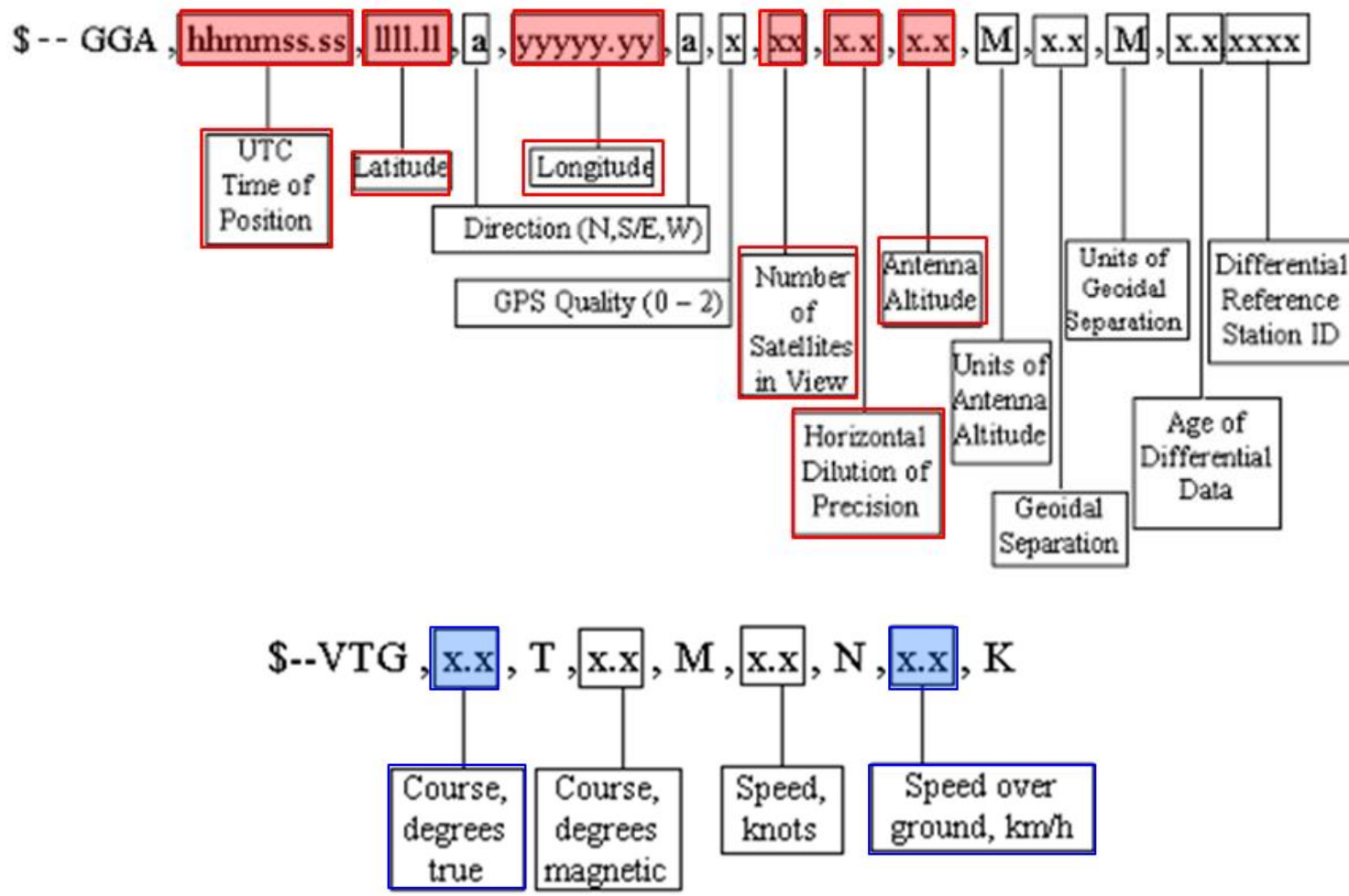

Figure 1. Morphology of NMEA strings: GGA (a) and VTG (b).

Due to the fact that GGA strings (Fig. 1a) provide position and time

197 simultaneously, instant velocity may be derived from a sequence of consecutive points.

198 However, it is important to keep in mind that this way of calculating speed differs from

199 the way speed is calculated in VTG strings (Fig 1b), as direct calculations of speed are

200 based on Doppler measurements, and are normally superior to position-based

201 calculations. The procedure to derive heading and speed (in a plane) from a sequence of

202 consecutive points defined by GGA strings (Fig. 1a), previously transformed to the local

203 tangent plane, can be summarized as follows. All the points recorded in the field by the

204 GNSS receiver constitute a set $\boldsymbol{T}$ of mathematical objects defining the trajectory of a robot

205 in time and 2D (planar) space. The specific points of the trajectory used at a given moment

206 to calculate heading and speed form a subset $\boldsymbol{S}$ of $\boldsymbol{T}$. In a formal definition, let $\boldsymbol{T}=\{1,2$,

$207 \ldots, m\}$ be the set of the indices of points defining the trajectory of a robot in the field, and

208 Let $\boldsymbol{S}=\{1,2, \ldots, h+1\} \subset \boldsymbol{T}$ be the subset of the indices of points used in the calculation of heading or speed and forming the calculation matrix $\mathrm{M}_{\mathrm{s}}$. The calculation matrix $\mathrm{M}_{\mathrm{s}}$ 
210 composed from the elements of $S$ will be dynamic - i. e. changing with time- and its

211 dimension will be $h+1 \times 3$. A general formulation of the calculating matrix for any given

212 point $k$ is shown in Eq. 1, where $k$ is the point in the sequence $\boldsymbol{T}$ being calculated, $\mathrm{E}$ is the

213 east coordinate $(\mathrm{m}), \mathrm{N}$ is the north coordinate $(\mathrm{m})$, and $t$ is the receiver time (s). The size

214 ( $m$ ) of set $\boldsymbol{T}$ depends on the traveling speed and the length of the trajectory traced by the

215 robot in the field, both imposed by each particular task. Therefore, the size of $\boldsymbol{T}$ is

216 generally determined by external factors. However, the size of subset $S$ is an important

217 parameter setup beforehand, which in practice influences the estimation of heading and

218 velocity from GNSS positions.

$$
M_{s}(k)=\left[\begin{array}{ccc}
E_{k} & N_{k} & t_{k} \\
E_{k-1} & N_{k-1} & t_{k-1} \\
E_{k-2} & N_{k-2} & t_{k-2} \\
\bullet & \bullet & \bullet \\
\bullet & \bullet & \bullet \\
\bullet & \bullet & \bullet \\
E_{k-h} & N_{k-h} & t_{k-h}
\end{array}\right]_{h+1 \times 3} \quad ; k>h ; t_{k}>t_{k-1}>\ldots>t_{k-h}
$$

219 The analysis of a point sequence $\boldsymbol{T}$ belongs to discrete mathematics, which focus

220 on mathematical structures that are discrete in nature and whose components can be

221 enumerated by integers, constituting a countable set that can be finite or infinite. The set

$222 \boldsymbol{T}$ that defines the robot's trajectory can be saved by the onboard computer and therefore

223 is finite and bounded by $m$. Consequently, the subset $S$ will also be finite and limited to

$224 h+1$ elements. The estimation of heading and velocity from a sequence of points requires

225 operating with finite differences, a technique within the scope of discrete calculus.

226 Velocity is a 2D vector whose norm is the magnitude of the robot forward speed, and

227 whose relation between vector components provides an estimate of the heading. The

228 calculation of velocity implies the use of differentiation, but with discrete data, there is a need to apply difference equations, which replace differentiation by taking the difference 
230 between adjacent terms to approximate differential equations. In a Cartesian coordinate

231 system such as the local tangent plane, where the abscissa axis (unit vector $\vec{i}$ ) represents

232 east and the ordinate axis (unit vector $\vec{j}$ ) represents north, the position vector $\overrightarrow{P_{k}}$ of a 233 point $k$ is given by Eq. 2 .

$$
\overrightarrow{P_{k}}=E_{k} \vec{i}+N_{k} \vec{j} \quad[\mathrm{~m}]
$$

The vector function distance $\vec{L}(k, \delta)$ of Eq. 3 provides the Euclidean distance between two points of $S$ whose indices are separated in the sequence by the calculation

236 step $\delta$. The reference point is $k$ and the calculation interval moves back $\delta$ positions in 237 matrix $\mathrm{M}_{\mathrm{s}}(\mathrm{k})$. Notice that necessarily $\delta<\mathrm{h}$, and $\vec{L}(k, \delta)$ is the backward difference 238 equation of position vector $\overrightarrow{P_{k}}$. The velocity vector for step $\delta$ is given in Eq. 4 .

$$
\begin{gathered}
\vec{L}(k, \delta)=\overrightarrow{P_{k}}-\overrightarrow{P_{k-\delta}}=\left(E_{k}-E_{k-\delta}\right) \vec{i}+\left(N_{k}-N_{k-\delta}\right) \vec{j} \quad[\mathrm{~m}] \\
\vec{V}(k, \delta)=\frac{\vec{L}(k, \delta)}{t_{k}-t_{k-\delta}} \quad\left[\mathrm{m} \cdot \mathrm{s}^{-1}\right]
\end{gathered}
$$

240 from $\vec{V}(k, \delta)$ through Eqs. 5 and 6 involving the inner product (dot product) of vectors,

241 the norm of a vector, and the trigonometric function arctangent.

$$
\begin{gathered}
V(k, \delta)=\|\vec{V}(k, \delta)\|=\sqrt{\vec{V}(k, \delta) \bullet \vec{V}(k, \delta)}=\frac{\sqrt{\left(E_{k}-E_{k-\delta}\right)^{2}+\left(N_{k}-N_{k-\delta}\right)^{2}}}{t_{k}-t_{k-\delta}} ; t_{k} \neq t_{k-\delta} \\
H(k, \delta)=\operatorname{arctg} \frac{E_{k}-E_{k-\delta}}{N_{k}-N_{k-\delta}} ; \quad N_{k} \neq N_{k-\delta} ; \quad m \geq k>\delta
\end{gathered}
$$




\section{Experimental design and setup}

A standard tractor was used to reproduce the robot trajectories necessary to accomplish this research. The tractor was equipped with two GNSS receivers setup for providing equivalent NMEA strings. The main receiver was a StarFire iTC ${ }^{\mathrm{TM}}$ differential

GPS (Deere \& Co, Moline, Il, USA) with capabilities to receive licensed signals SF2 and

248 free signals SF1, although all the tests were conducted with the free signal SF1 as most

249 of the local users in Southern Europe are not willing to pay for signal fees. For this

250 situation (SF1), the static accuracy is $75 \mathrm{~cm}$ and the pass to pass accuracy is $\pm 33 \mathrm{~cm}$. In

251 addition, a secondary low-cost receiver (Garmin $18 \mathrm{x}-5 \mathrm{~Hz}$, Olathe, Ks, USA) with an

252 advertised error inferior to $3 \mathrm{~m}$ was installed in the cabin of the tractor.

The goal of the experimental design is the acquisition of objective data to help in the design process of robot navigation with regards to GNSS information. In particular, the outcomes of the experiments are meant to rank the parameters of the first row in Table 1. As the use of two receivers per robot is not considered, comparisons were established between GGA-based positions, GGA-based heading and speed, and VTG-based heading (course) and speed over ground. Specific software was developed and customized to the needs of this research, recording GGA and VTG strings simultaneously from both receivers. This software application implemented several filtering routines to enhance the reliability of GNSS data described in detail in Rovira-Más and Banerjee (2013). The heading definition of Eq. 6 is mathematical rather than computational, which in practice

263 leads to stability problems when divisions by zero occur and heading estimates do not 264 vary smoothly in the entire range $\{0,360\}^{\circ}$. In order to compare heading calculations with direct measurements from VTG messages, Eq. 6 was amplified by the logic set in Table 2. 
Condition for membership

$\mathbf{H}(\mathbf{k}, \delta)\left[{ }^{\circ}\right]$

\begin{tabular}{cc}
\hline $\mathrm{E}_{\mathrm{k}} \geq \mathrm{E}_{\mathrm{k}-\delta} \& \mathrm{~N}_{\mathrm{k}}>\mathrm{N}_{\mathrm{k}-\delta}$ & $\frac{180}{\pi} \cdot \operatorname{arctg} \frac{E_{k}-E_{k-\delta}}{N_{k}-N_{k-\delta}}$ \\
\hline $\mathrm{E}_{\mathrm{k}}>\mathrm{E}_{\mathrm{k}-\delta} \& \mathrm{~N}_{\mathrm{k}}=\mathrm{N}_{\mathrm{k}-\delta}$ & 90 \\
\hline $\mathrm{E}_{\mathrm{k}} \leq \mathrm{E}_{\mathrm{k}-\delta} \& \mathrm{~N}_{\mathrm{k}}>\mathrm{N}_{\mathrm{k}-\delta}$ & $360-\frac{180}{\pi} \cdot \operatorname{arctg} \frac{E_{k-\delta}-E_{k}}{N_{k}-N_{k-\delta}}$ \\
\hline $\mathrm{E}_{\mathrm{k}}<\mathrm{E}_{\mathrm{k}-\delta} \& \mathrm{~N}_{\mathrm{k}}=\mathrm{N}_{\mathrm{k}-\delta}$ & $180+\frac{180}{\pi} \cdot \operatorname{arctg} \frac{E_{k-\delta}-E_{k}}{N_{k-\delta}-N_{k}}$ \\
\hline $\mathrm{E}_{\mathrm{k}} \leq \mathrm{E}_{\mathrm{k}-\delta} \& \mathrm{~N}_{\mathrm{k}}<\mathrm{N}_{\mathrm{k}-\delta}$ & $90+\frac{180}{\pi} \cdot \operatorname{arctg} \frac{N_{k-\delta}-N_{k}}{E_{k}-E_{k-\delta}}$ \\
\hline $\mathrm{E}_{\mathrm{k}} \geq \mathrm{E}_{\mathrm{k}-\delta} \& \mathrm{~N}_{\mathrm{k}}<\mathrm{N}_{\mathrm{k}-\delta}$ & 180 \\
\hline $\mathrm{E}_{\mathrm{k}}=\mathrm{E}_{\mathrm{k}-\delta} \& \mathrm{~N}_{\mathrm{k}}<\mathrm{N}_{\mathrm{k}-\delta}$
\end{tabular}

The detailed analysis of the first row of Table 1 unfolds in the set (or family) of 270 tests listed in the first column of Table 3, each of them comprising a number of experiments focused on a determined parameter. Field tests were conducted in 2010, 2011, and 2012, and several farm plots at three different locations were used to acquire the data, one of them barren and all the others planted with grapevines.

Table 3. Experimental design.

Parameter evaluated

\begin{tabular}{|c|c|}
\hline Parameter evaluated & \multirow{2}{*}{$\begin{array}{c}\text { Alternatives studied } \\
\text { Low-cost receiver }\end{array}$} \\
\hline \multirow{2}{*}{ Receiver type } & \\
\hline & High-performance receiver \\
\hline \multirow{2}{*}{ NMEA string type } & GGA-based Heading \& Speed \\
\hline & VTG-based Course \& Ground Speed \\
\hline \multirow{2}{*}{$\mathrm{M}_{\mathrm{s}}(\mathrm{k})$ size for speed calculation } & $h=9 \rightarrow \operatorname{Dim}\left(\mathrm{M}_{\mathrm{s}}(\mathrm{k})\right)=[10 \times 3]$ \\
\hline & $h=5 \rightarrow \operatorname{Dim}\left(\mathrm{M}_{\mathrm{s}}(\mathrm{k})\right)=[6 \times 3]$ \\
\hline \multirow{2}{*}{$\begin{array}{l}\text { Threshold velocity for heading } \\
\text { calculation }\end{array}$} & $\mathrm{V}=3 \mathrm{~km} / \mathrm{h}$ \\
\hline & $\mathrm{V}=5 \mathrm{~km} / \mathrm{h}$ \\
\hline \multirow{4}{*}{$\mathrm{M}_{\mathrm{s}}(\mathrm{k})$ size for heading calculation } & Slow motion $(\mathrm{V}<5) \rightarrow h=15$ \\
\hline & Fast motion $(\mathrm{V} \geq 5) \quad \rightarrow \quad h=31$ \\
\hline & Slow motion $(\mathrm{V}<5) \rightarrow h=9$ \\
\hline & Fast motion $(\mathrm{V} \geq 5) \quad \rightarrow \quad h=21$ \\
\hline
\end{tabular}

According to Table 3, the first parameter under study is the influence of the

277 receiver specifications on the final outcomes. Normally, high-performance receivers

278 behave more reliably and stably than low-cost devices, but they are not free of external errors such as signal blockage or multipath reflections. This family of tests is devoted to 
280 find out the role that embedded quality indices HDOP (horizontal dilution of precision)

281 and number of satellites play in the early detection of corrupted data. As shown in Table

282 1, the key navigation parameters heading and speed can be directly read from VTG

283 strings, and alternatively calculated from position and time available in GGA strings. The

284 second set of experiments tries to elucidate if both estimations are really equivalent in

285 terms of precision and reliability, i. e., if they are complementary or redundant. The

286 calculation of heading from GGA positions depends on the forward speed of the robot,

287 and consequently it is important to establish which is the optimal size of $\mathrm{Ms}(\mathrm{k})$ for the

288 estimation of speed; a large matrix will introduce a considerable delay, but a small one

289 will yield noisy outcomes. The set of experiments in the third row of Table 3 tries to

290 determine the reasonable size for the speed matrix. The stability of heading calculation

291 according to forward speed (Van Bergeijk et al., 1998; Anderson and Bevly, 2010)

292 advises to adapt the initial equation (Eq. 6) to the speed, but a threshold speed is necessary

293 to classify the motion of the robot according to speed. As farm robots cannot move very

294 fast in off-road terrains, two regimens of low and high speed were established. The fourth

295 set of experiments looks for the boundary limit between high and low speed. Once a limit

296 speed has been fixed, the last family of experiments investigates the size of the sequence

297 of points that best estimates the robot course in real time. As the straight application of

298 Eq. 6 and Table 2 introduces significant noise, a combination of four partial headings

299 with different weights was implemented in the experimental vehicle. Figure 2 illustrates

300 the concept of partial headings for a generic calculation matrix of $h$ elements, and Eq. 7

301 provides a practical example with $h=32$ of how partial headings may be weighted in the 302 algorithm. 


$$
\left.\begin{array}{ll}
H_{\delta 1} \stackrel{\text { def }}{=} & \delta=31:[k-31, k] \\
H_{\delta 2} \stackrel{\text { def }}{=} & \delta=9:[k-9, k] \\
H_{\delta 3} \stackrel{\text { def }}{=} & \delta=22:[k-31, k-9] \\
H_{\delta 4} \stackrel{\text { def }}{=} & \delta=7:[k-12, k-5]
\end{array}\right\} \quad H(x)=\frac{2 \cdot H_{\delta 1}+H_{\delta 4}}{3}
$$

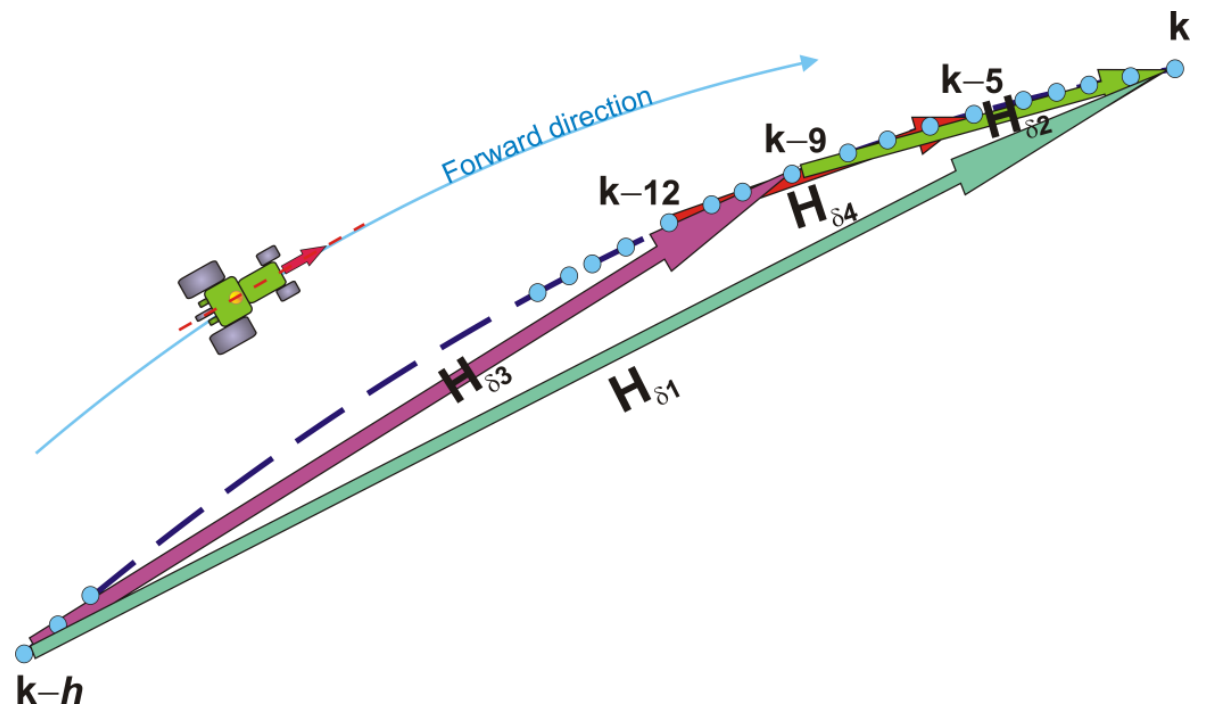

Figure 2. Partial headings in a generic sequence of $h$ elements.

\section{Results}

The hypotheses set in Table 3 were tested in the field over three seasons at a

307 variety of conditions. A summary of the empirical consequences found along the representative plots.

\begin{tabular}{ccc}
$\begin{array}{c}\text { Parameter } \\
\text { Evaluated }\end{array}$ & Field observation & $\begin{array}{c}\text { Related } \\
\text { figure }\end{array}$ \\
\hline $\begin{array}{c}\text { Receiver } \\
\text { Quality }\end{array}$ & $\begin{array}{c}\text { Helpful but not definitive as fail-safe strategy } \\
\text { HDOP and number of satellites not sufficient to grant stability }\end{array}$ & 3 \\
\hline $\begin{array}{c}\text { NMEA string } \\
\text { Type }\end{array}$ & $\begin{array}{c}\text { At startup; VTG is superior and GGA is slow } \\
\text { At headland; GGA is superior and VTG yields wrong estimates }\end{array}$ & 4 \\
\hline $\begin{array}{c}\text { Dim }\left(\mathrm{M}_{\mathrm{s}}(\mathrm{k})\right) \\
\text { for speed }\end{array}$ & $\begin{array}{c}\text { Positive results around } h=9 ; \\
h<9 \text { is less stable and } h>9 \text { is too slow }\end{array}$ & 5 \\
\hline $\begin{array}{c}\mathrm{V}_{\text {threshold }} \\
\text { for heading }\end{array}$ & $5 \mathrm{~km} / \mathrm{h}$ is more stable and accurate than $3 \mathrm{~km} / \mathrm{h}$ & 6 \\
\hline $\begin{array}{c}\text { Dim }\left(\mathrm{M}_{\mathrm{s}}(\mathrm{k})\right) \\
\text { for heading }\end{array}$ & $\begin{array}{c}\text { Reduced } \mathrm{M}_{\mathrm{s}}(\mathrm{k}) \text { responds faster with negligible initial delay; } \\
\text { however, it cannot be adopted for stability reasons }\end{array}$ & 7 \\
\hline
\end{tabular}


The experimental vehicle was setup to simultaneously record the same trajectories

314 using both GNSS receivers (Garmin and StarFire). The objective of this set of tests was

315 to determine the influence of low-precision positioning on vehicle states, especially

316 heading. Figure 3a plots a typical example of low-cost positioning in comparison with the

317 estimates of an accurate GNSS receiver, where errors oscillate in the range 0-10 m. The

318 impact of this positioning imprecision on the calculation of headings is quantified in

319 Figure $3 b$.
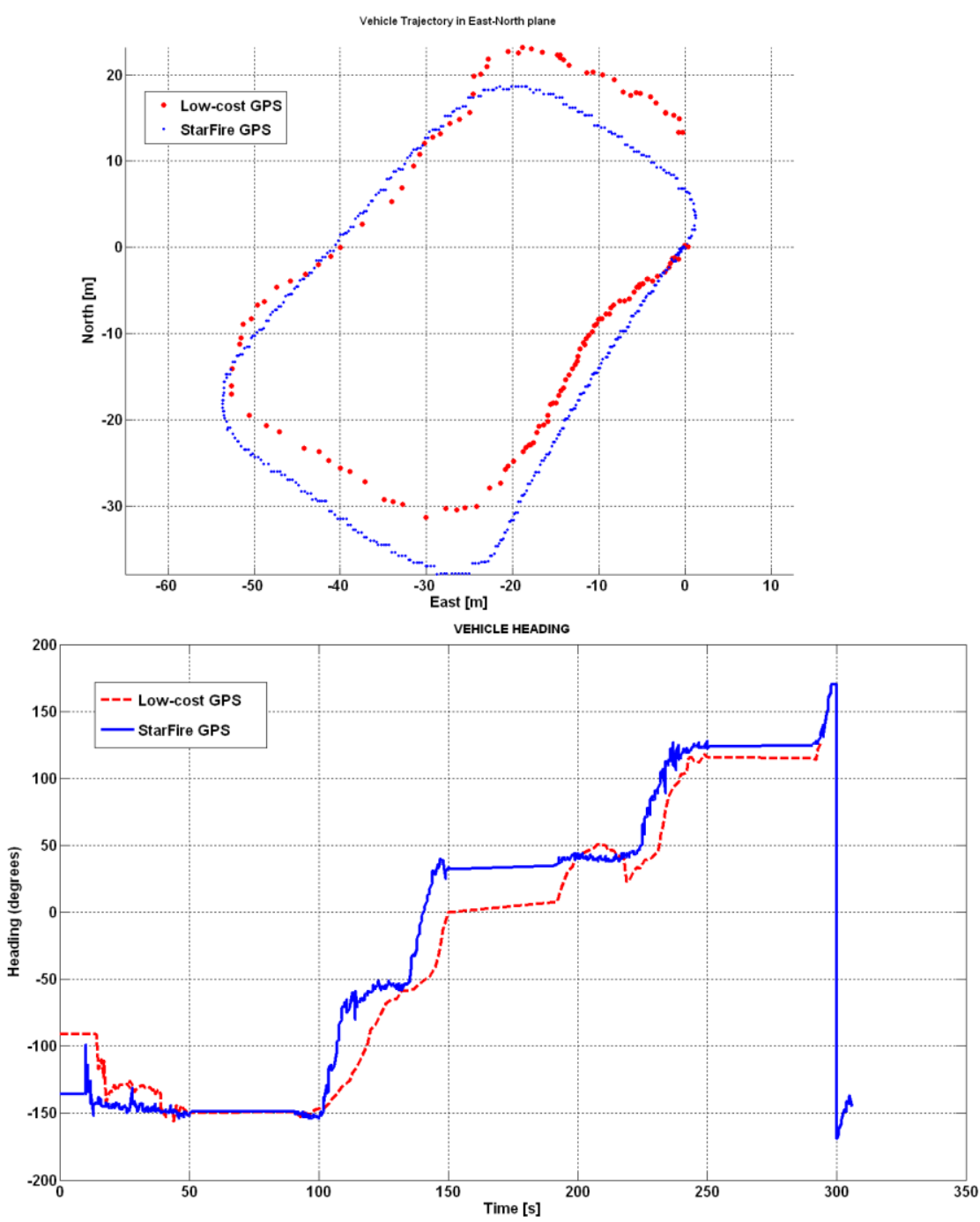

Figure 3. Comparison of receiver performance: east-north plot of tested trajectory (a) and vehicle heading (b).

The measurement of vehicle heading and speed - principal states for robot 
two different techniques: course and Doppler-based velocity contained in VTG strings of the NMEA code; and alternatively heading and forward velocity computed on a sequence of points whose positions are carried in GGA strings of the NMEA code. Figure 4 shows the comparison for two representative tests conducted in different days; a 6-row trajectory

330 recorded in the evening (Fig. 4a) and a 10-row trajectory registered the morning of the

331 following day. Potential local effects on signal quality were considered by repeating the experiments in different days or time of the day from early morning to dusk.

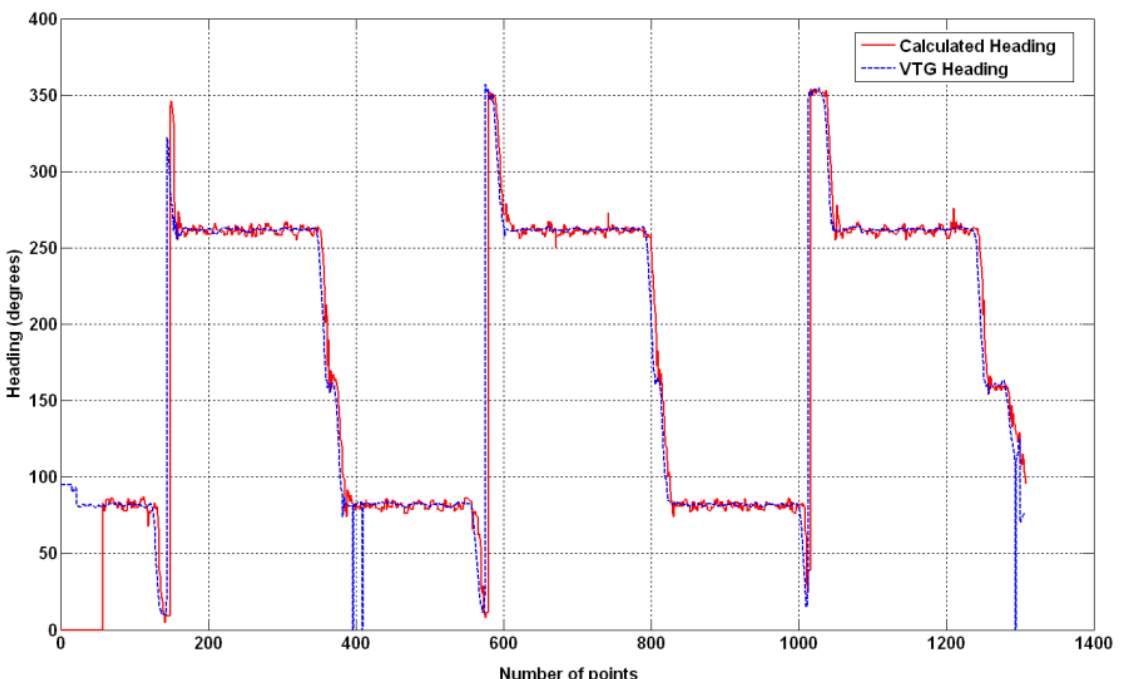

(a)

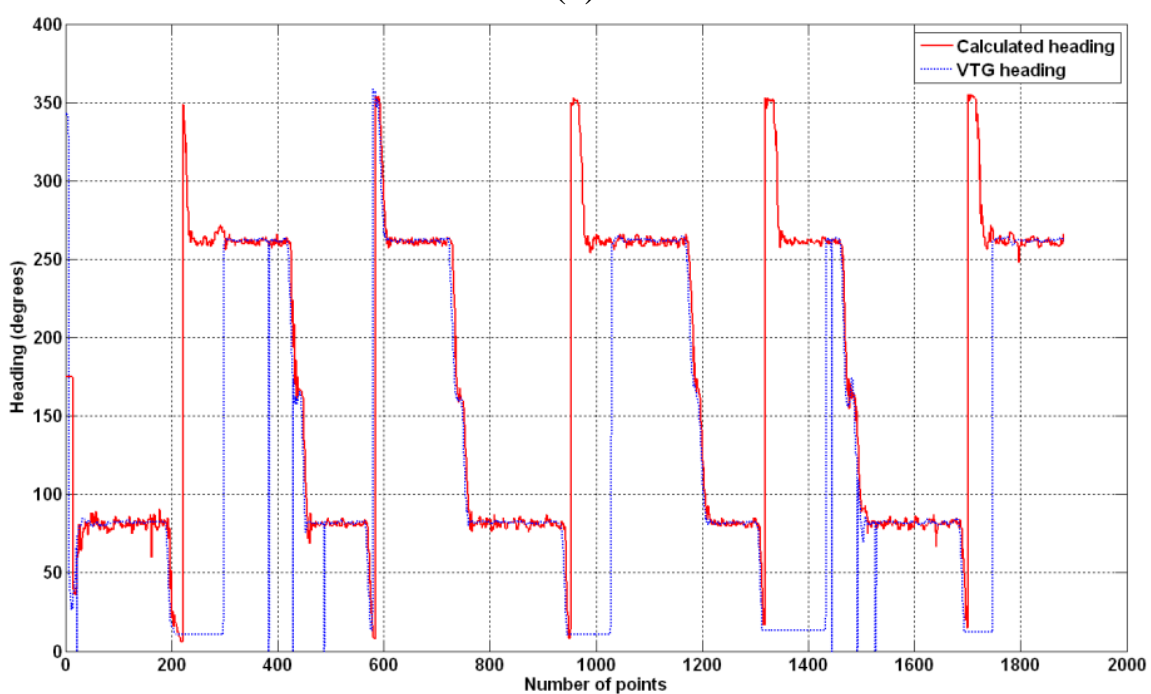

(b)

Figure 4. Comparison of heading read from VTG-based course and calculated from GGA-based positions: 6-row evening test (a) and 10-row morning test (b).

Fixing the optimal size of the matrix $\mathrm{M}_{\mathrm{s}}(\mathrm{k})$ used to calculate speed was key for 
341 course and velocity. Figure 5 shows the results of downgrading the dimension of $\mathrm{M}_{\mathrm{s}}(\mathrm{k})$

342 from ten points to six points for estimating a target velocity around $4 \mathrm{~km} / \mathrm{h}$, usual for a

343 farm robot. VTG measurements are also provided as a ground-truth reference after

344 checking their coincidence with the vehicle's speedometer included in the dashboard.

345 Notice that the number of points evaluated is slightly different as a consequence of

346 different speeds; Fig. 5a plots a faster run and therefore less points were recorded as the

347 GPS frequency was always $5 \mathrm{~Hz}$.

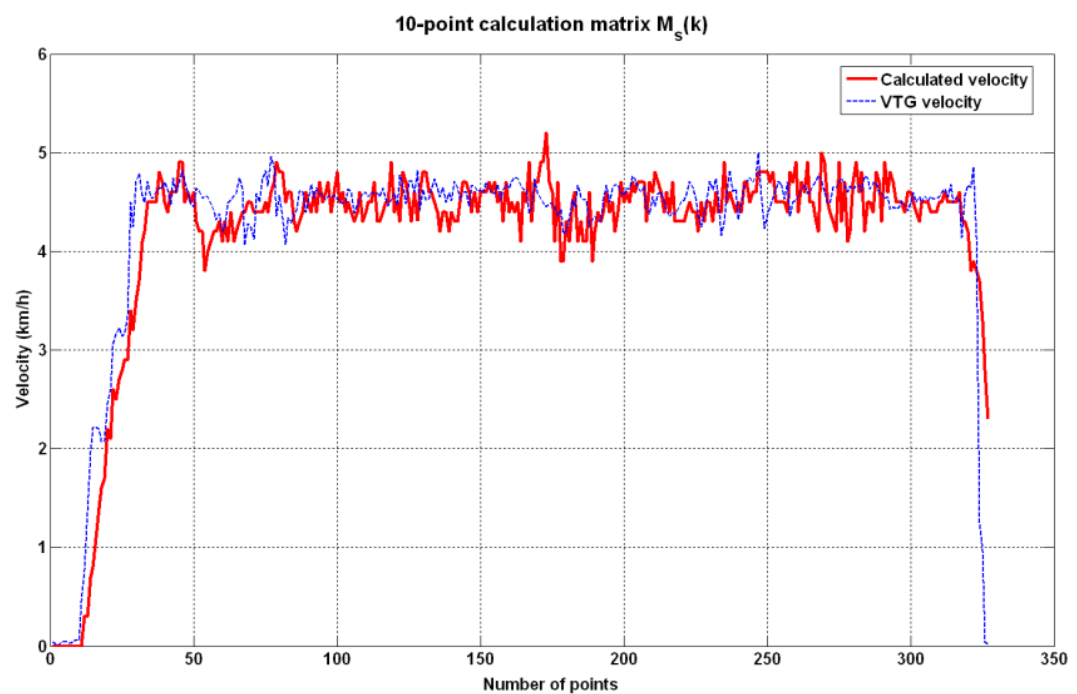

(a)

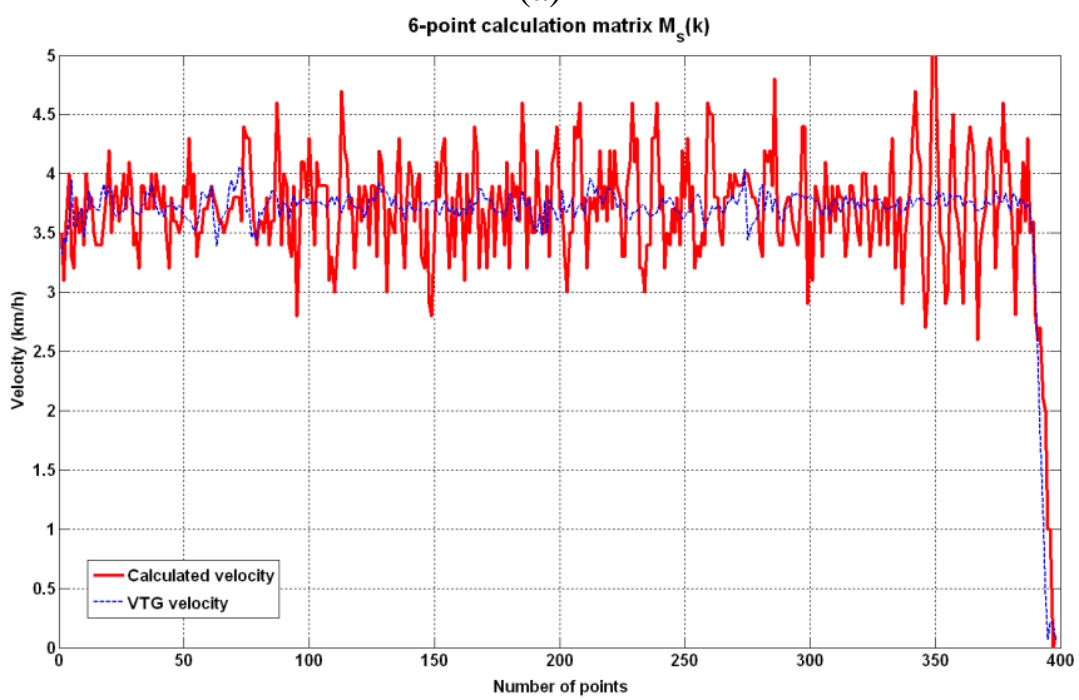

(b)

Figure 5. Influence of calculation matrix dimensions on speed: 10-point matrix (a); and 6-point matrix (b).

The calculation of heading from a sequence of points depends on the robot's 
356 speed. The range of speeds at which farm robots need to operate is limited, normally in

357 the range $0-10 \mathrm{~km} / \mathrm{h}$. For this situation, it is sufficient to consider two speed ranges,

358 namely slow and fast motion, but the critical point is determining the threshold that

359 classifies the speed of the robot in one of these two classes. Figure 6 depicts the results

360 of the tests devoted to study the size of subset $S$ for the speed calculation matrix, in

361 particular it shows the effects of lowering the threshold speed from $5 \mathrm{~km} / \mathrm{h}$ (Fig. $6 \mathrm{~b}$ ) to 3

$362 \mathrm{~km} / \mathrm{h}$ (Fig. 6a) on heading estimations for a target forward velocity of $3 \mathrm{~km} / \mathrm{h}$, very likely

363 in a farm robot. As in previous cases, the VTG course is provided as a reference.

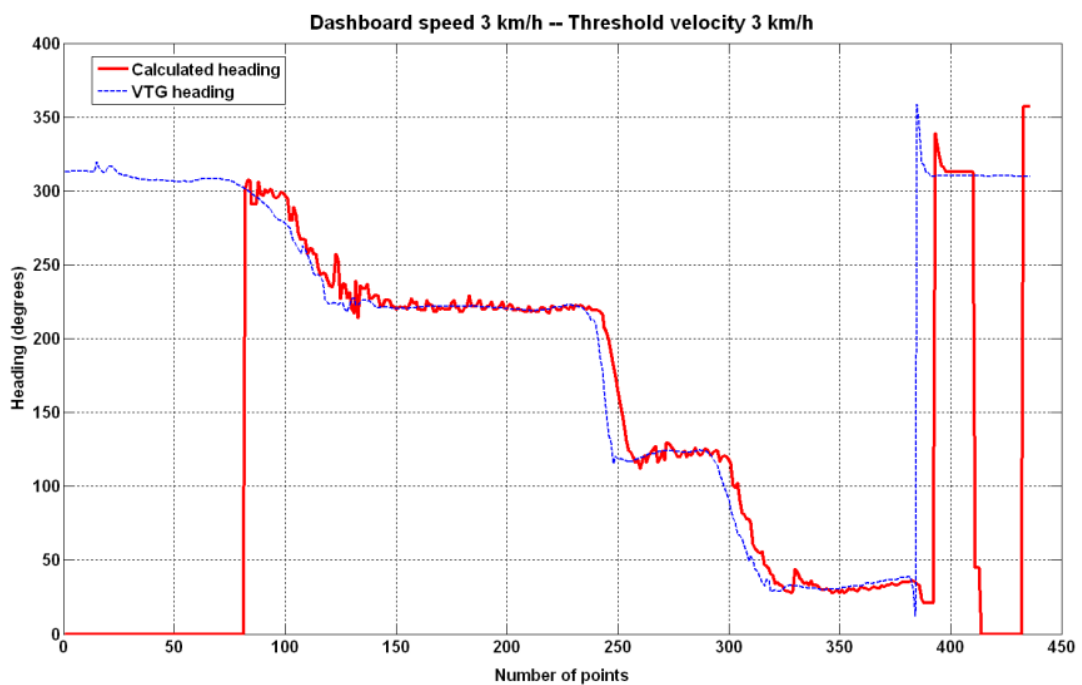

(a)

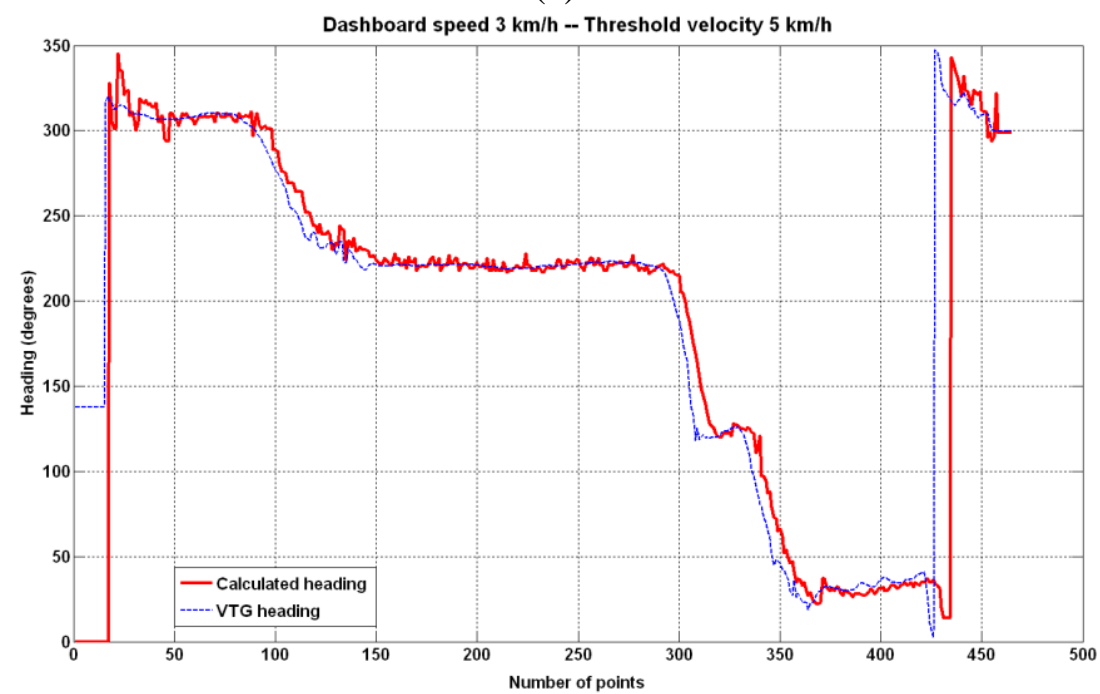

(b)

Figure 6. Influence of threshold velocity $V_{t}$ on the calculation of robot heading: $V_{t}=3$ $\mathrm{km} / \mathrm{h}(\mathrm{a})$; and $\mathrm{V}_{\mathrm{t}}=5 \mathrm{~km} / \mathrm{h}(\mathrm{b})$. 
The last analysis of this work focused on the dimensions of the calculation matrices for heading estimation, which was different for slow motion and for fast motion according to the selected threshold velocity $(5 \mathrm{~km} / \mathrm{h})$. The actual size of the calculation matrices determines the particular definition of partial headings $\mathrm{H}_{\delta \mathrm{i}}$ as illustrated in Fig. 2. Field experience showed that for the range of vehicle speeds considered and a GNSS frequency of $5 \mathrm{~Hz}$, the dimensions proposed in Table 3 provided a general description of the problem. Figure 7 plots the heading calculated when the vehicle moves at $4 \mathrm{~km} / \mathrm{h}$ and the calculation matrix has 22 points for slow motion and 10 points for fast motion. This plot may be compared with those of figures 3,4 , and 6 which implement matrices of 32 and 16 points for the calculation of headings.

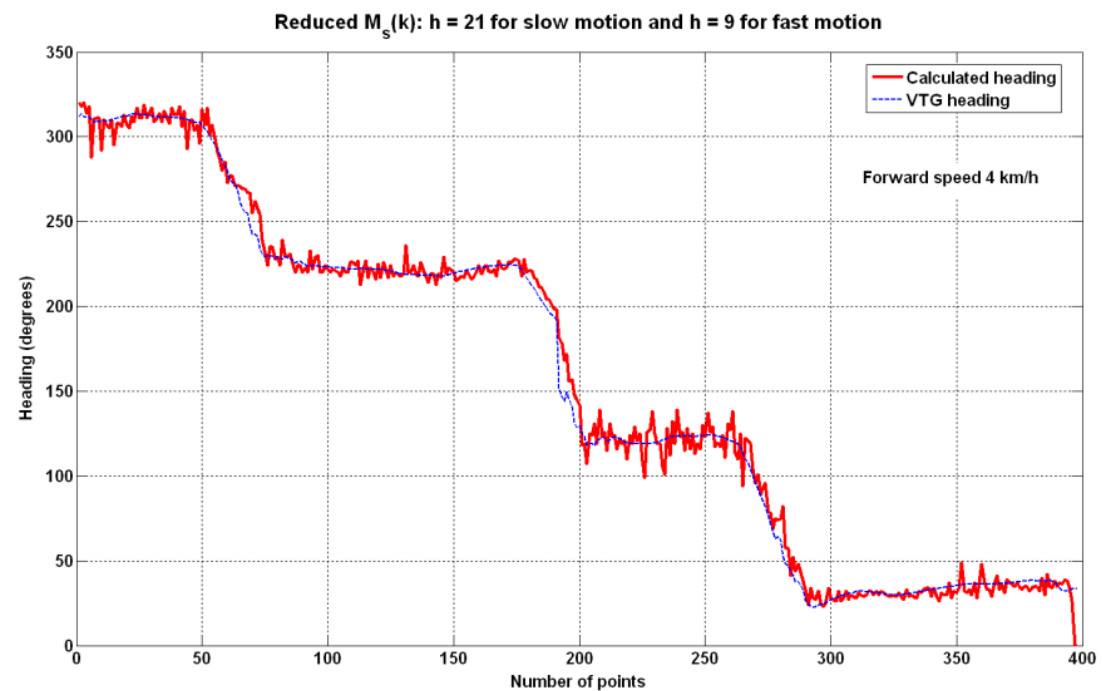

380

381

382

383
Figure 7. Heading calculated with $h=\{21,9\}$ and forward speed of $4 \mathrm{~km} / \mathrm{h}$.

\section{Discussion}

The comparison of receivers led to expected results such as the degradation of the trajectory caused by significant positioning errors, but it also revealed interesting phenomena. In particular, the StarFire trajectory of Fig. 3a shows the east-west drift already noticed by Rovira-Más and Banerjee (2013), and the quality indicators number of satellites and HDOP were surprisingly better for the low-cost receiver (10 satellites versus 9 and HDOP $0.8-1$ versus constant 1), which in practical terms means that 
embedded indices are not completely reliable and must be weighted by the quality of each

390 receiver; in other words, low-cost receivers are usually not aware of their inaccurate

391 outputs. In addition, isolated strings of corrupted data were detected over long periods of

392 operation for both receivers, and therefore signal corruption and blockage induced by

393 external conditions such as tall trees and surrounding buildings are always a risk to take

394 into account. Nevertheless, the average performance of the high quality receiver was

395 clearly superior as evidenced by the heading estimation of Fig. 3b, which in reality only

396 yields useful headings for the StarFire system. In light of these results, the selected

397 receiver should be that of highest standards within the assumed cost-efficiency policy,

398 but it will not grant long-run stability unless filtering routines and quality checks are

399 implemented and continuously executed in the navigation engine of the robot.

400 Furthermore, a sophisticated receiver will not grant stability either because multipath

401 reflections, poor constellations, and signal blockages will eventually generate outliers that

402 might create a risk for the navigation of the robot if a contingency plan has not been

403 considered.

404 The fact that heading and velocity can be obtained from the same receiver

405 alternatively from two sources and using different techniques, amplifies the usability of

406 GNSS for robot navigation, as course and speed are basic parameters for setting

407 navigation strategies. The point is whether it makes sense calculating heading and speed

408 from a sequence of points if they are already available in VTG strings of the form

409 established in Fig. 1b. To find it out, several trajectories were followed with the

410 simultaneous recording of heading and speed obtained from both methods. The

411 experimental field consisted of parallel rows of vines spaced $3 \mathrm{~m}$ and $130 \mathrm{~m}$ long, with a

412 constant heading of $83^{\circ}$ when the vehicle traveled east (forward trip) and $263^{\circ}$ when

413 traveling west in the return trips. Figure 4a shows the results of the evening test, where 
414 VTG measurements locked the right course from the beginning of the track whereas the

415 calculated headings were delayed in time and did not yield the right course until 50 points

416 were processed. However, the VTG course produced erroneous estimates in the vicinity

417 of point 400 , which coincided with a headland turn. A close inspection of embedded

418 quality indices around point 400 reveals a drop in satellites from 9 to 6 together with an

419 increase in HDOP from 1.2 to 2.7. These values for the indices are considered acceptable

420 and do not fully justify the VTG heading drop at the headland. Figure $4 \mathrm{~b}$ shows the results

421 of a longer test performed in the same scenario the following day, with different

422 atmospheric conditions and ephemeris. The degradation of VTG heading at some - but

423 not all- headland turns is more apparent than in Fig. 4a, and shows that GGA estimates

424 were much more stable than VTG ones. These outcomes are not totally unexpected,

425 because the GNSS accuracy dependency on the travel direction due to satellite geometry

426 was already reported by Wu et al. (2006), and according to Standard X587 (ASABE,

427 2010), a loss of signal is more common in agriculture at the edge of the fields, and

428 consequently the Standard recommends conducting Dynamic Signal Reacquisition tests

429 on the U-turns of standardized travel courses, reporting heading and speed accuracy

430 separately for straight segments and curved segments. The failures detected at the

431 headland turns with VTG heading (course) and speed advised the redundant estimation

432 of GGA-based navigation states, and justified the analysis of the size of $\mathrm{M}_{\mathrm{s}}(\mathrm{k})$ for

433 calculating heading and speed as well as the selection of a threshold velocity between

434 slow and fast motion, discussed in detail in the following paragraphs.

435 Once the convenience of calculating navigation parameters from a sequence of 436 trajectory points has been proved, the size of the sets of points (subset $\boldsymbol{S}$ ) used in the 437 calculation must be determined as it will impact the final outcomes. The dimension of the 438 calculating matrix will be different for speed, slow motion heading, and fast motion 
439 heading, being speed especially important because it determines the size of the calculation

440 matrices used for the estimation of heading. Extensive testing revealed that a calculation

441 matrix of ten rows $(h=9)$ resulted in reasonable estimates. However, the initial delay

442 could be eliminated with a smaller matrix allowing for faster cycles. To check such

443 assumption, speed estimation was carried out with a reduced matrix of six elements $(h=$

444 5), as compared in Fig. 5. For common speeds of approximately $4 \mathrm{~km} / \mathrm{h}$, Fig 5a shows

445 that $h=9$ produces more stable outcomes than a matrix considering six points (Fig. 5b).

446 Heading calculations require the application of different equations according to

447 forward speed, which in practical terms implies the selection of three basic parameters:

448 the threshold speed that separates slow motion from fast motion, and the dimensions of

449 the calculation matrices for each type of motion, which in turns leads to a differentiated

450 definition of partial headings as generally indicated in Fig. 2. Being $4 \mathrm{~km} / \mathrm{h}$ a reasonable

451 operating speed for a farm robot, two boundary speeds of $3 \mathrm{~km} / \mathrm{h}$ and $5 \mathrm{~km} / \mathrm{h}$ were

452 analyzed as thresholds between the slow motion class and the fast motion class. Fig. 6

453 shows greater stability for the $5 \mathrm{~km} / \mathrm{h}$ threshold (6b), especially at initiation where the

454 lower threshold is affected by a significant delay (6a). For both estimates there exists a

455 slight delay with respect to VTG values, which become very resourceful as a redundant 456 check to measure the stability of calculated heading. The attenuation of noise in the

457 calculation of heading required the use of large matrices, which eventually resulted in 458 important delays, mainly if compared to VTG estimates. Although satisfactory results 459 were achieved with sequences of 32 elements in fast motion and 16 elements in slow 460 motion, if the same response could be granted with a significant reduction in the size of 461 the sequence, such response would be faster and more promptly available. To prove so, 462 the fast motion matrix was downgraded to 22 elements whereas the slow motion matrix 463 was reduced to 10 points. Unfortunately, Fig. 7 indicates that for a usual speed of $4 \mathrm{~km} / \mathrm{h}$, 
464 the reduction in the number of elements for estimating heading leads to less stable results

465 when compared to figures 3,4 , and 6 , where headings were calculated with the larger

466 matrices. The results found along the experiments, and summarized in Table 4, were

467 implemented in the experimental vehicle according to the block diagram of Fig. 8. Notice

468 that for this application, speeds below $0.5 \mathrm{~km} / \mathrm{h}$ were disregarded as noise, and speed

469 discrimination occurred at $5 \mathrm{~km} / \mathrm{h}$. This flow chart provides a practical example of the

470 role of GNSS in the navigation strategies of farm robots, but other specific applications

471 will likely require other definition and combination of partial headings, or calculation

472 matrices of different dimensions, even though the philosophy is valid for a wide variety

473 of agricultural moderate-size robots.

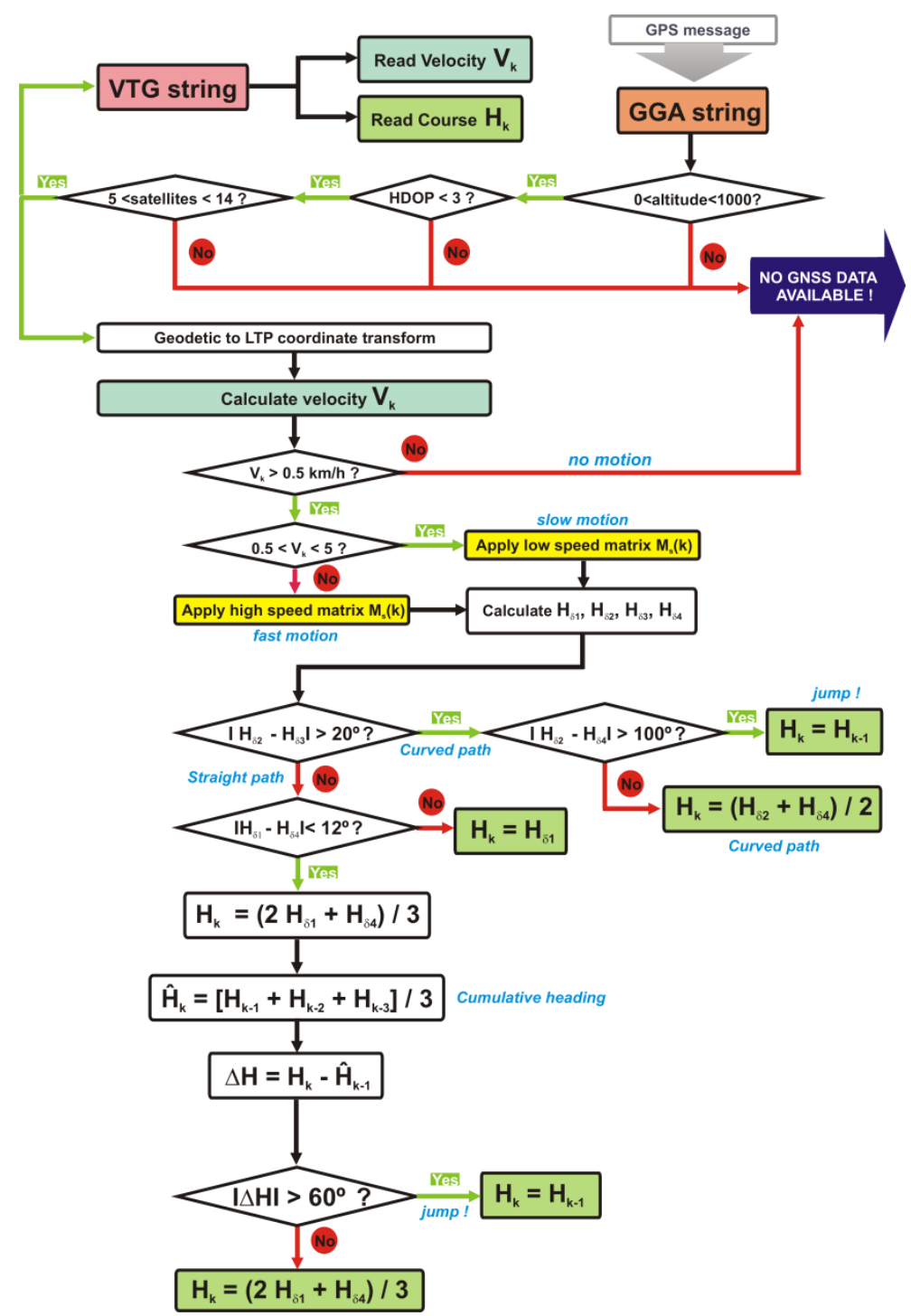


Figure 8. Block diagram of GNSS algorithm implemented in the vehicle.

476

477

478

480

481

482

484

\section{Conclusions}

This research intended to outline the role of GNSS in cost-efficient farm robots, especially in terms of navigation. Results showed that it is always convenient to integrate GNSS in this kind of robots, but this integration can never be plug-and-play; on the contrary, it requires the careful filtration of data in such a way that spurious information never enters the processing loop of the navigation engine. The accuracy of the GNSS receiver has a positive impact in the mobility of the robot, but it cannot grant long-term stability in agricultural fields where multipath reflections and signal blockage are unpredictable. As a result, GNSS-borne data requires redundancy and continuous failsafe checking. This redundancy may be achieved both within GNSS data and from external sensors. The former implies the concurrent analysis of VTG and GGA strings whereas the latter requires the implementation of alternative sensors such as compasses, radars, or optical devices. The experiments conducted showed that GGA and VTG information is complementary rather than substitutive, given that VTG course and speed is accurate at startup but becomes unreliable at the headland turns where GGA-derived heading and speed is stable. Nevertheless, in order to get accurate heading and speed from a sequence of points defined by GGA strings, important decisions must be made regarding the primary parameters of the calculation algorithm. In particular, the size of the calculation matrices and the strategy to deduce stable estimates from a sequence of points defining the robot's path is essential to make the most efficient use of GNSS. Overall, GNSS is a key component of farm robots for the cost-efficient redundant information that it provides. The conceptual scheme of Fig. 9 summarizes the role of GNSS in farm robots, with the redundant estimation of critical navigation parameters. Dashed lines in pale ink represent a potential augmentation of the navigation system as long as it is compatible 
500 with the assumed cost-efficiency policy. There exists a substantial difference between

501 cost-efficiency robots and low-cost GNSS. Whether low-cost GNSS devices can help

502 with the design of cost-effective agricultural robots is a relevant question, and the key is

503 on reliability rather than cost as long as cost-efficiency requirements are met. As a result,

504 RTK devices and subscription fees will not be helpful in general terms, but the ideal cost

505 for the receiver will depend on the final cost fixed for the robot and the safety standards

506 established for the tasks programmed in the robot. The smart implementation of GNSS

507 will definitely result in the smart behavior of agricultural robots.

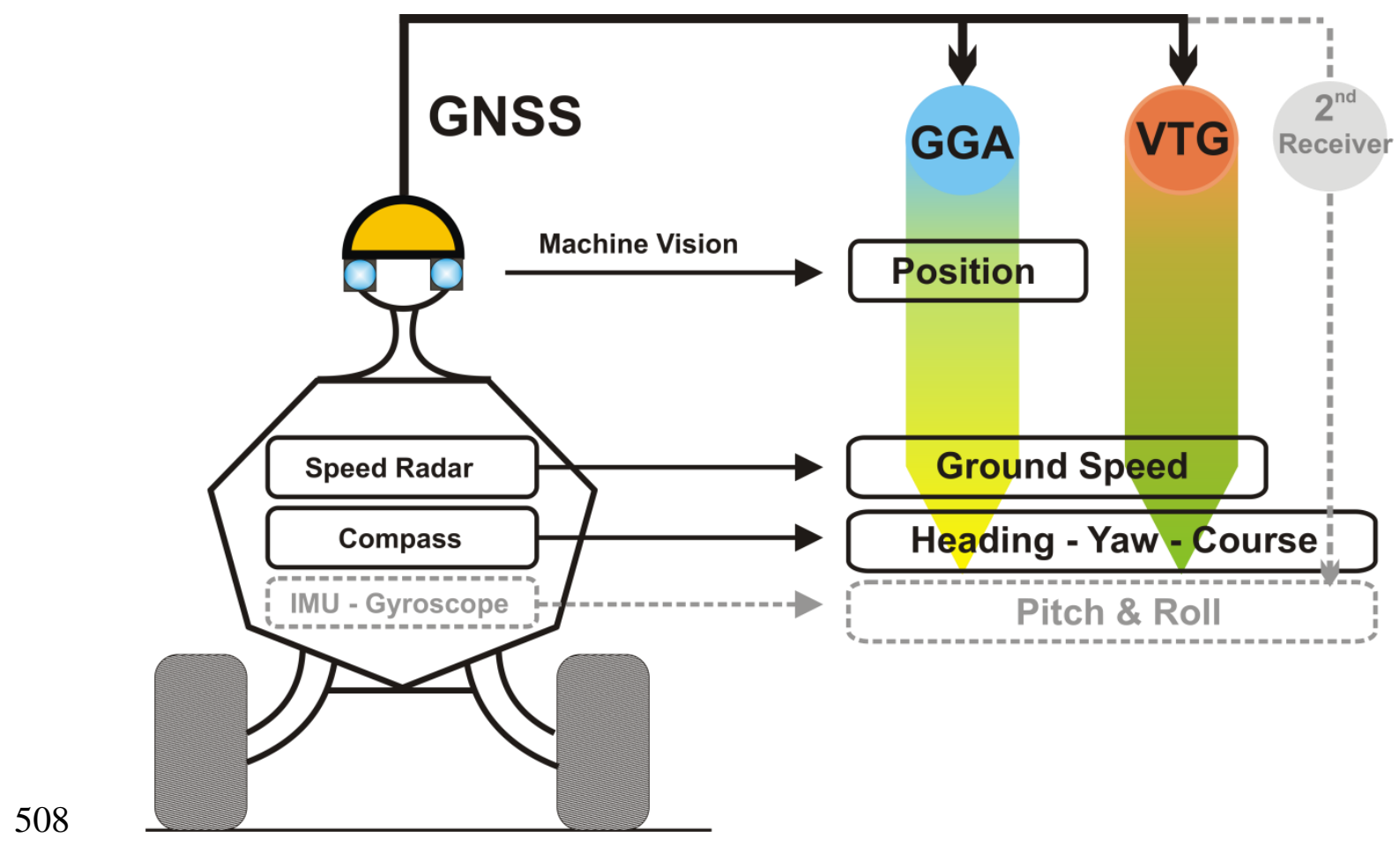

Figure 9. Role of GNSS in the navigation engines of farm robots.

$510 \quad$ References

511 Anderson, R., Bevly, D. M., 2010. Using GPS with a model-based estimator to estimate

$512 \quad$ critical vehicle states, Vehicle System Dynamics 48 (12): 1413 - 1438.

513 ASABE, 2010. Dynamic testing of satellite-based positioning devices used in agriculture.

514 Standard X587 (Draft 10), Available from http://elibrary.asabe.org. [Accessed on

$515 \quad 3$ December 2012] 
516 Chosa, T., Omine, M., Itani, K., 2007. Dynamic performance of global positioning system velocity sensor for extremely accurate positioning, Biosyst. Eng. 97: 3-9.

518 Cocco, L., Rapuano, S., 2007. Accurate speed measurement methodologies for Formula One cars, in: Proc. IEEE Instrumentation and Measurement Technology Conference, Warsaw, Poland.

Ding, W., Wang, J., 2011. Precise velocity estimation with a stand-alone GPS receiver. Journal of Navigation 64: 311-325.

Emmi, L., González-de-Soto, M., Pajares, G., González-de-Santos, P., 2014. Integrating Sensory/Actuation Systems in Agricultural Vehicles. Sensors 14(3): 4014-4049.

Joerger, M., Pervan, B., 2006. Autonomous ground vehicle navigation using integrated GPS and laser-scanner measurements, in: Proc. of Position, Location, and Navigation Symposium (PLANS), San Diego, CA, 2006, pp. 988-997.

Kondo, N., Monta, M., Noguchi, N., 2011. Agricultural Robots: Mechanism and Practices. Trans Pacific Press, Balwyn North Victoria, Australia.

Nørremark, M., Griepentrog, H. W., Nielsen, J., Søgaard, H. T., 2008. The development and assessment of the accuracy of an autonomous GPS-based system for intrarow mechanical weed control in row crops, Biosyst. Eng. 101: 396-410.

Rovira-Más, F., 2009. Recent innovations in off-road intelligent vehicles: in-field automatic navigation, Recent Patents on Mechanical Engineering 2: 169-178.

Rovira-Más, F., 2010. Sensor architecture and task classification for agricultural vehicles and environments. Sensors 10: 11226-11247; doi:10.3390/s101211226

537 Rovira-Más, F., Zhang, Q., Hansen, A. C., 2010. Mechatronics and Intelligent Systems for Off-road Vehicles. Springer, London, pp. 68 - 71.

Rovira-Más, F., Banerjee, R., 2013. GPS data conditioning for enhancing reliability of automated off-road vehicles, J. Automobile Eng. 227 (4): 78 - 92. 
541 Serrano, L., Kim, D., Langley, R. B., Itani, K., Ueno, M., 2004. A GPS velocity sensor:

542 how accurate can it be?- A first look, in: Proc. of ION National Technical

543 Meeting, San Diego, CA, 2004, pp. 875-885.

544 Van Bergeijk, J., Goense, D., Keesman, K. J., Speelman, L., 1998. Digital filters to

545 integrate global positioning system and dead reckoning. J. Agric. Eng. Res. 70:

$546 \quad 135-143$.

547 Wu, C., Ayers, P. D., Anderson, A. B., 2006. Influence of travel direction on GPS

548 accuracy for vehicle tracking, Transac. ASABE 49(3): 623 - 634.

549

550

551

552 